تأثير إضافة الكبريت في درجة حموضة التربة pH ونمو وإنتاجية نبات السلجم

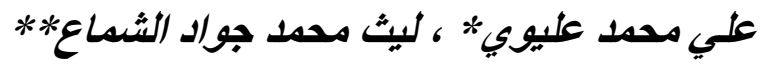

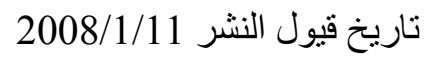

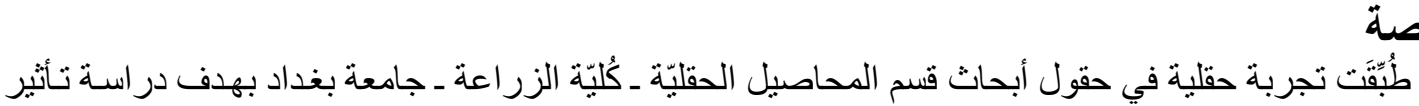

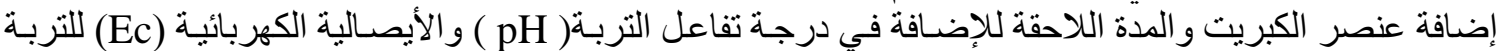

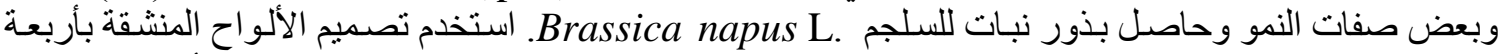

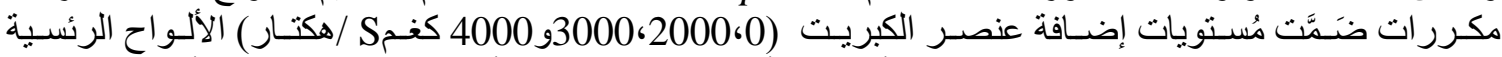

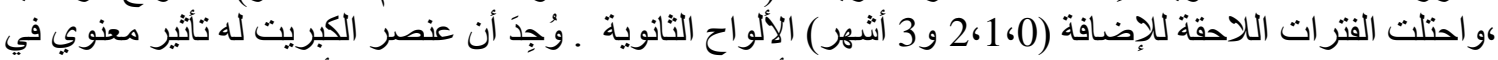

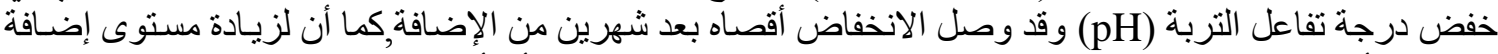

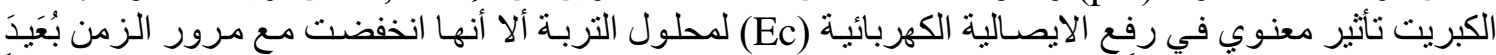
الإضافة. أثر الكبريت معنوياً في زيادة ارتفاع النبات النات الاانه خفض محتوى البذور من الزيت في حين لم يؤنثر معنوياً

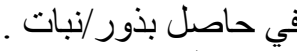

الَمُقدمة

اظهرت النتائج وجود تأثثير معنوي لإضـافة الكبريت

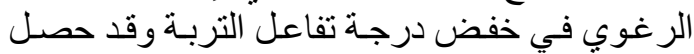

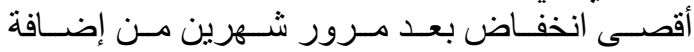
الكبريت عند مستوى(4000 كغتS / هكتـار), و وأدت

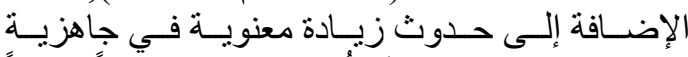

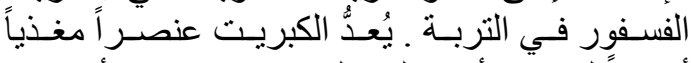

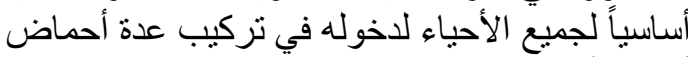

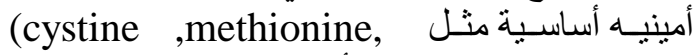
Biotin, Co(و عدة مر افقات أنزيمية مثلةcystiene) enzyme A, Thiamine pyrophosphate and Thioredoxine and فضـال عن (Lipoic acid (Sulpholipids كما يدخل الكبريت في تركيب كثير الإنير من المركبات غير الأساسية في النبات لكنها تسهر في في آلاعي

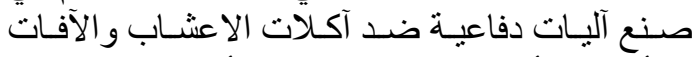

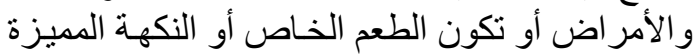

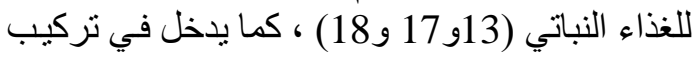
بعض الفيتامينات وفي عملية تركيب الكلوروفيل كليل لكنه

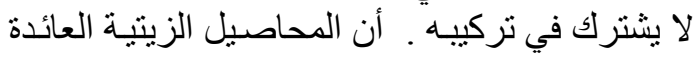

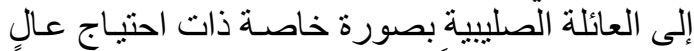

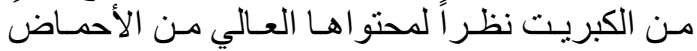

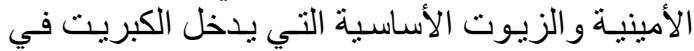
تركيبها( 15 و 26 ). إن المقدار المعار الملائم من الكبريت

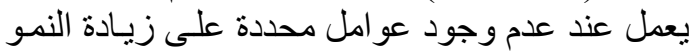
الخضري وزيادة أنتاج المادة الجافة عن طريق زئل زيادة

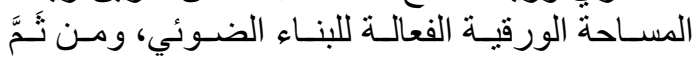

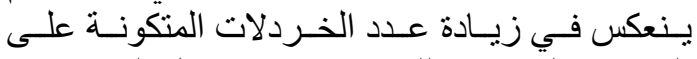

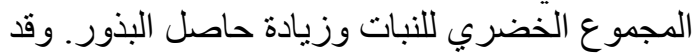

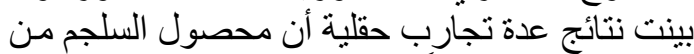

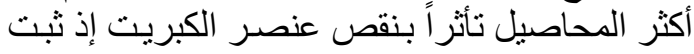

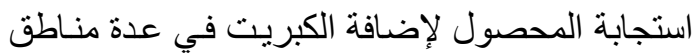
من المملكة المتحدة (23و 24 ).

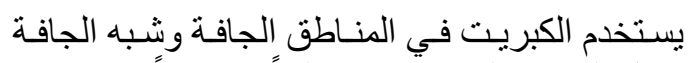

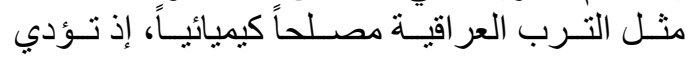

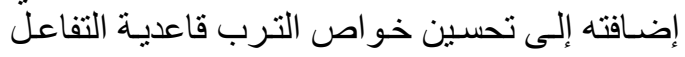

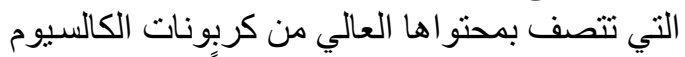

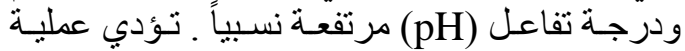
أكسدة عنصر الكبريت بعد أضافته إلى التربة وبة وبفعل

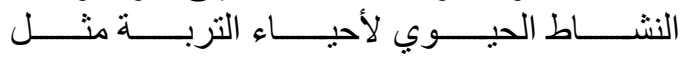

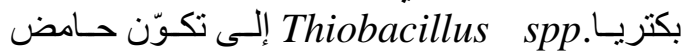

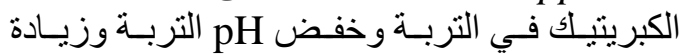

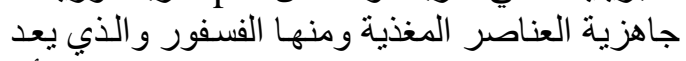

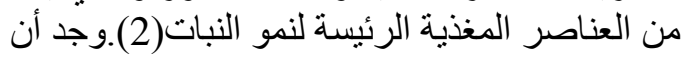

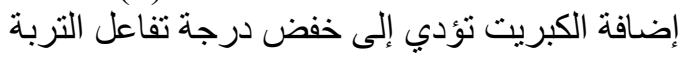

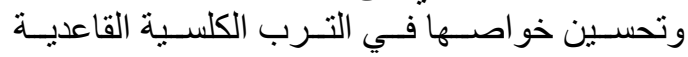

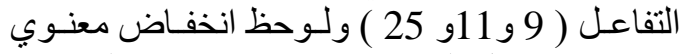
في درجة تفاعل التربة وزيادة في جاهزيـة العناصر

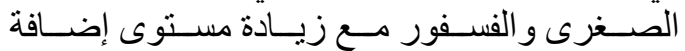

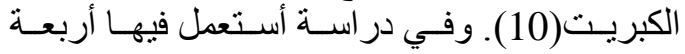

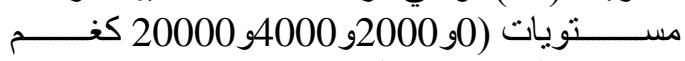

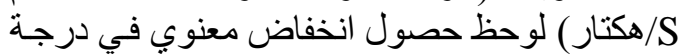

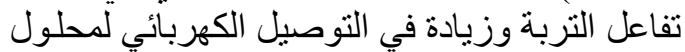

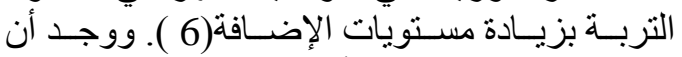
إضافة الكبريت إلى تربة أريزونا الإنا الكلسية بمسنويات

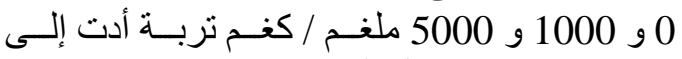

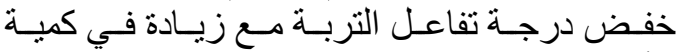

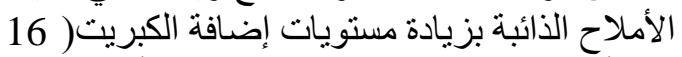

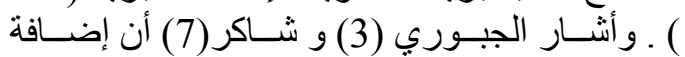

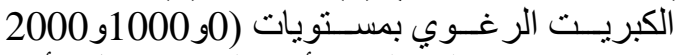

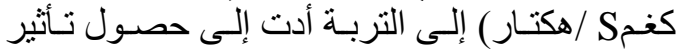

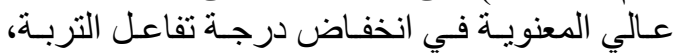

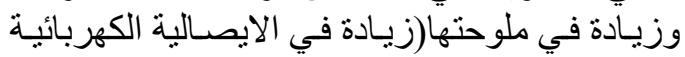

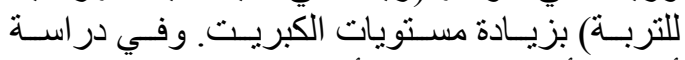

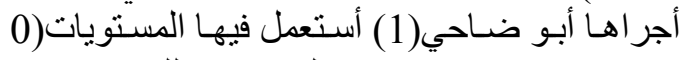
و2000 و4000 و6000 كيلو غر امS/ للهكتار ) 
2005-2004 على شكل خطوط بطول (3م)يفصـل بينها مسـافة(40سـم) بكثافـة نباتيـة بلغت 833330

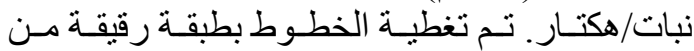

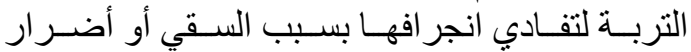

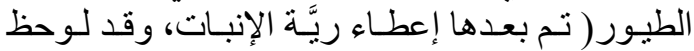

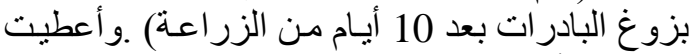

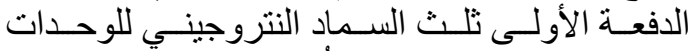

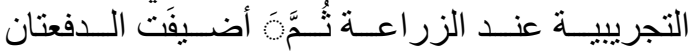

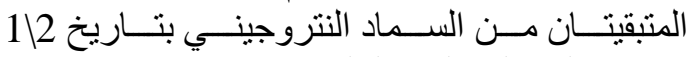

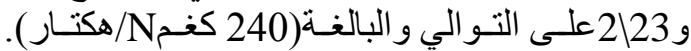
أجريت عملية خف النباتات عند ظهور أول ورقتتين

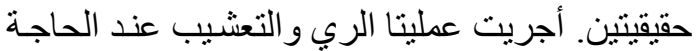

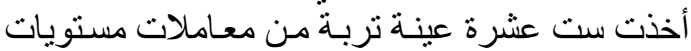
الكبريت الأربعة من المكررات الأربعة لدئ لدر اسـة تأثنير

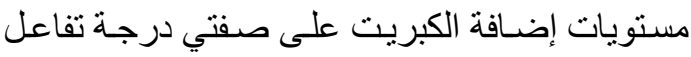

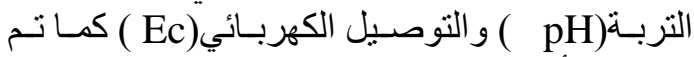

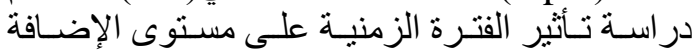

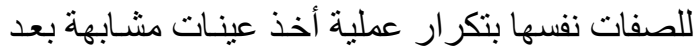

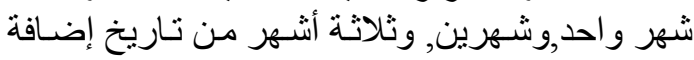

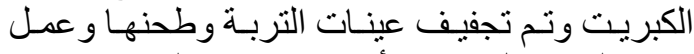

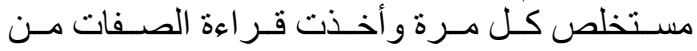

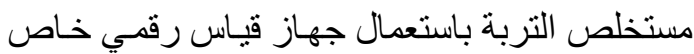

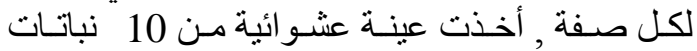

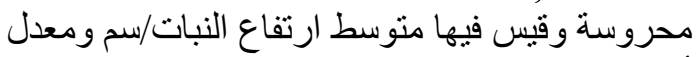

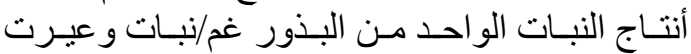

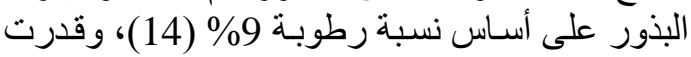
نسبة الزيت في البذور باستعمال جهاز

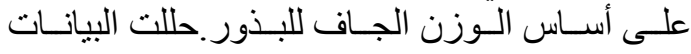

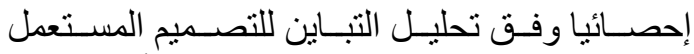

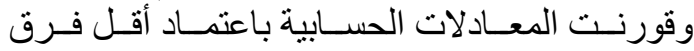
معنوي(L.S.D.) على مستوى احتمالية 5\% النتائج و المناقشئة

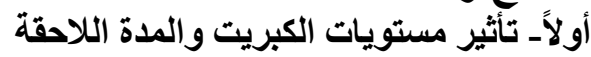

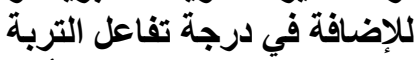

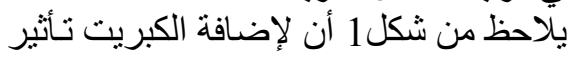

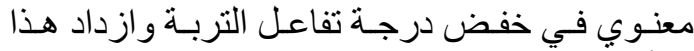

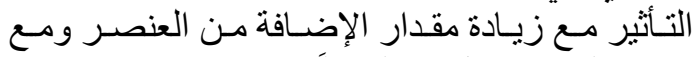

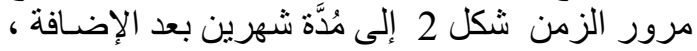

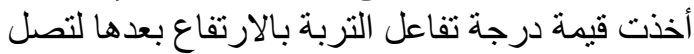

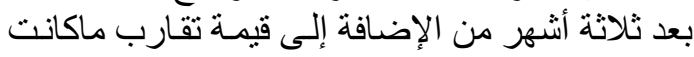

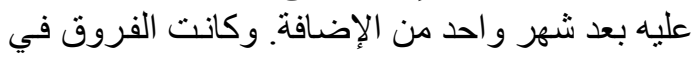

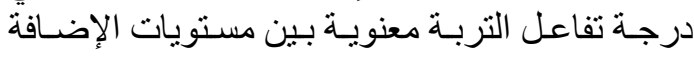

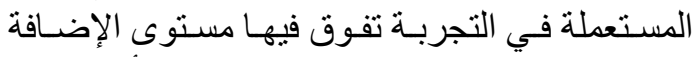

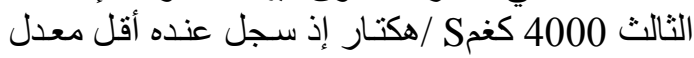

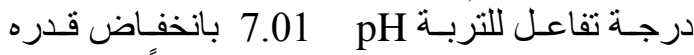
0.39عنه قبل الإضافة وكان الفربة الفرق معنوياً بين جميع

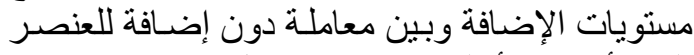

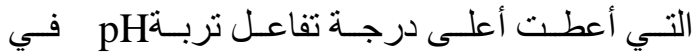

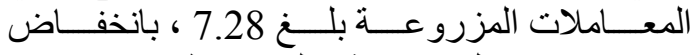

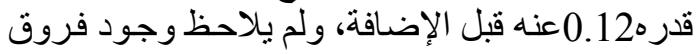

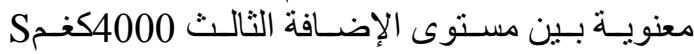

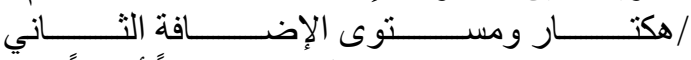
3000كغــ/هكتاروكـان الفـرق معنويـاً أيضـاً بـين الفين
أن إضافة ( 60 كغم /هكتار ) من الكبريت لم تعطي

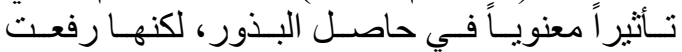

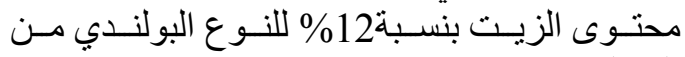

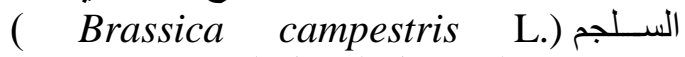
وبنسبة16\% لمحصول الخردل الهندي (Bnsica

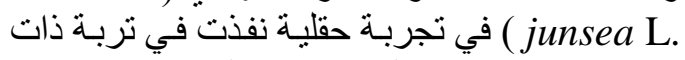

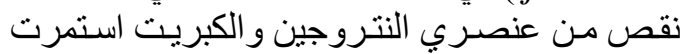

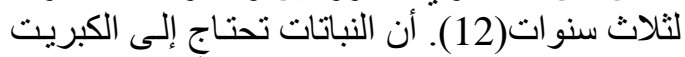

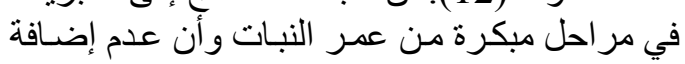

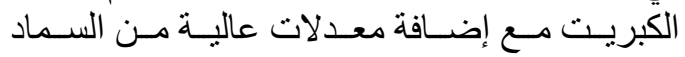

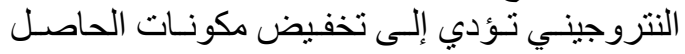

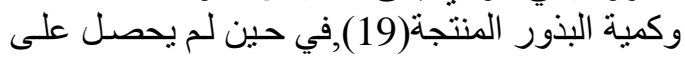

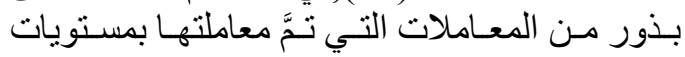

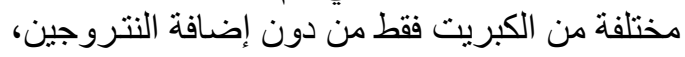

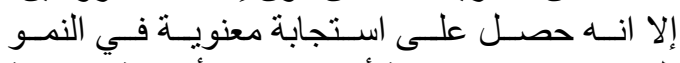

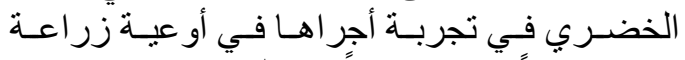

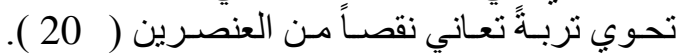

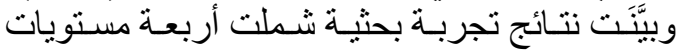

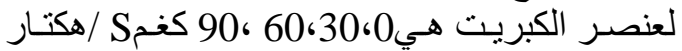
أن زيادة مستوى إضافة الكبريت تؤدي إلى استجابة

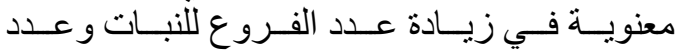

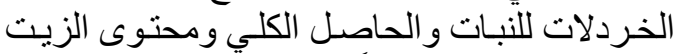

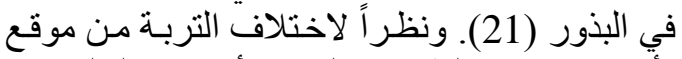

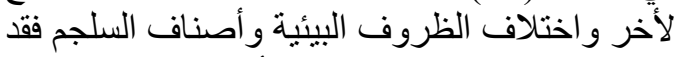

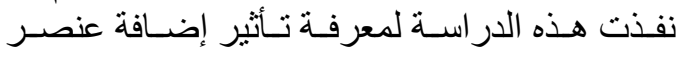

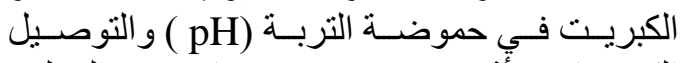

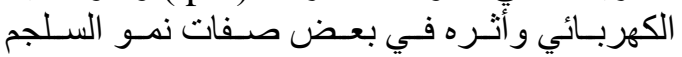
وحاصله من البذور.

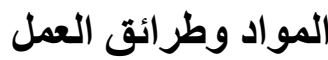

نفذت تجربة في حقل قسم المحاصيل الحقلية

كلية الزراعة/ أبو غريب بهربة فئ دراسة تأثير استعمال

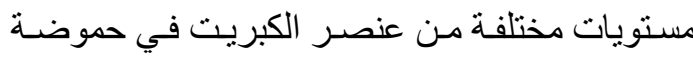

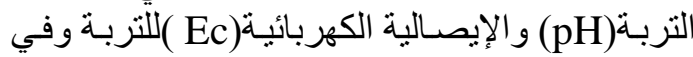
بعض صفات نمو نبات السلجم وحاصله من البذائل البذور صنف Pactol ـ أتبع تصميم الألو اح المنشقة ضدت التهن

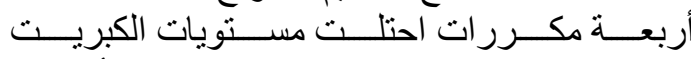

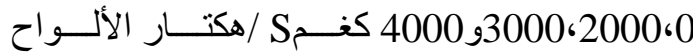
الرئيسية واحتلت الفتر ات اللاحقة للإضـافة 0، 1، 1،

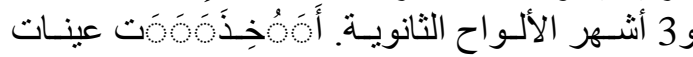

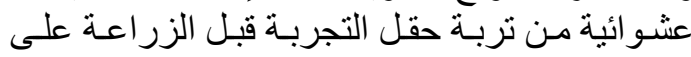

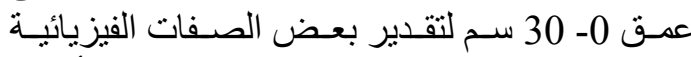

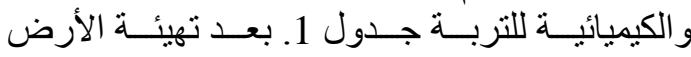

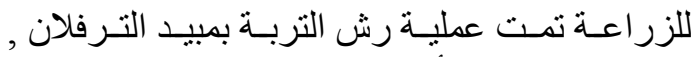

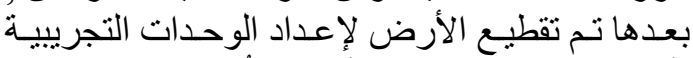

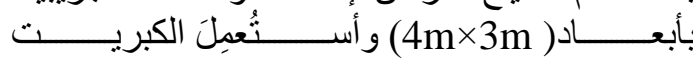

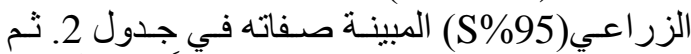

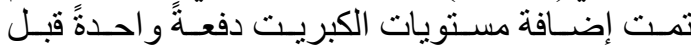

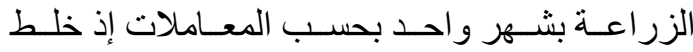

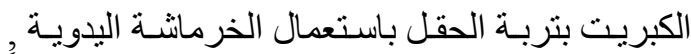

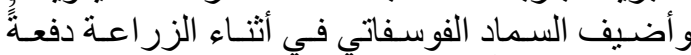

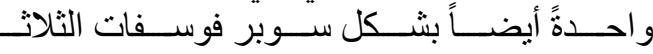

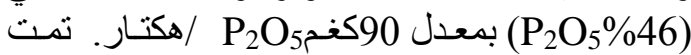
الزراعة في بداية تشرين الثاني خلال الموسم الثنتوي 


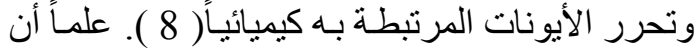

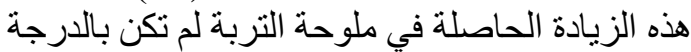

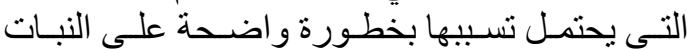

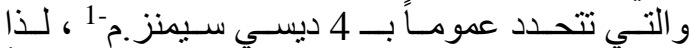

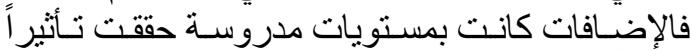

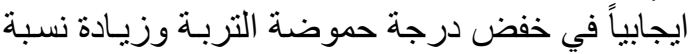
الأيونات الموجبة وربما سببت زيادة في في ملوحة التربة التربة

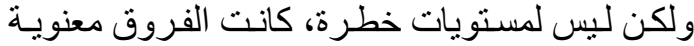

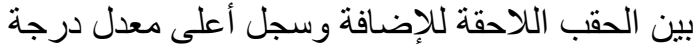

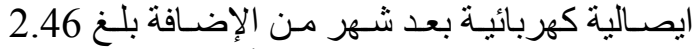

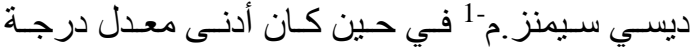

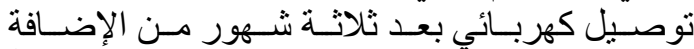

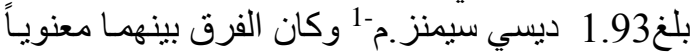

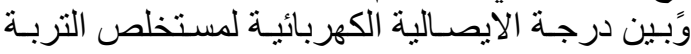

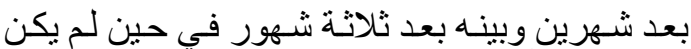

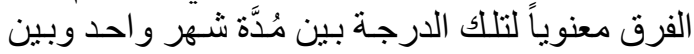

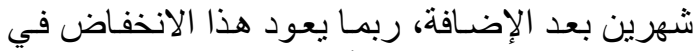

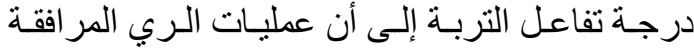

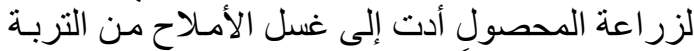

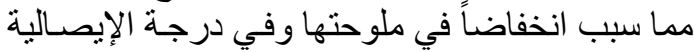

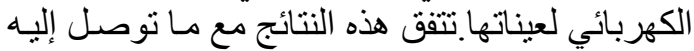

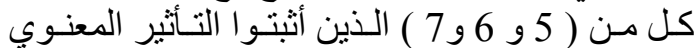

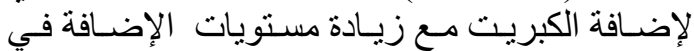

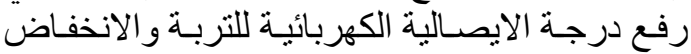
في هذه الدرجة في الترب المزروعة مع مرور الزمن بعيد الإضافة. ثُأثاً تأثير مستويات الكبريت في بعض صفات نبات

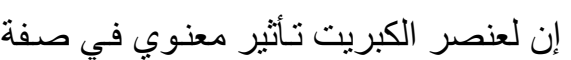

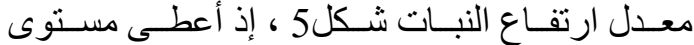

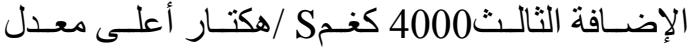

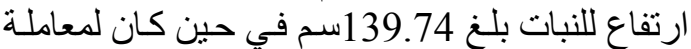
من دون ألإضافة أقل معدل ارتفاع نبات بلغ 132.25

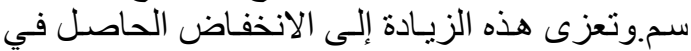

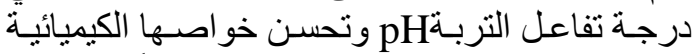

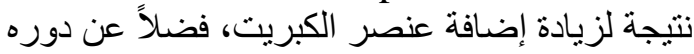

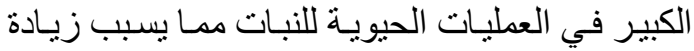

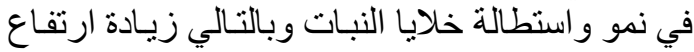

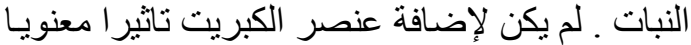
في معدل انتاج البذور للنبات شكل 6. 5.

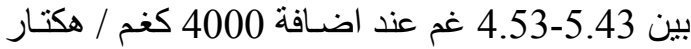

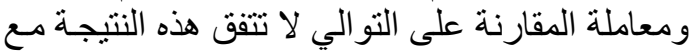

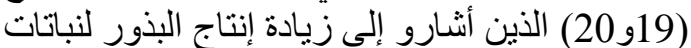

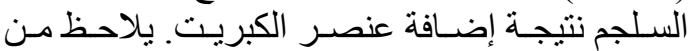

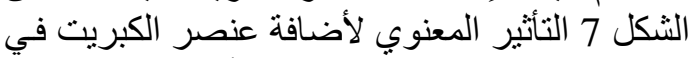

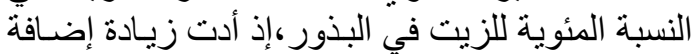

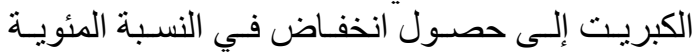

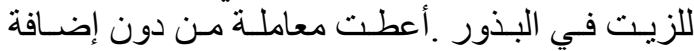

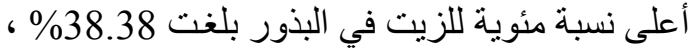

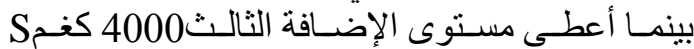

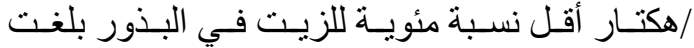

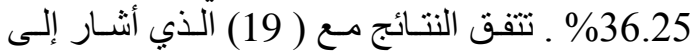

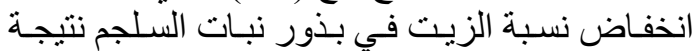

مستوى الإضافة الثاني و الثالث وبين مستوى الإضافة

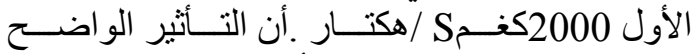

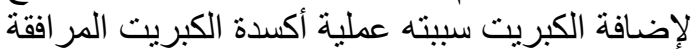

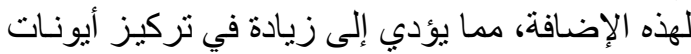

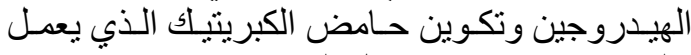

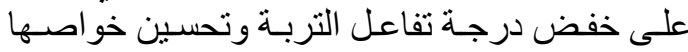

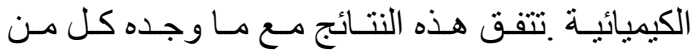

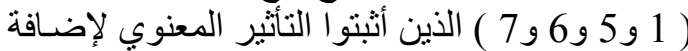

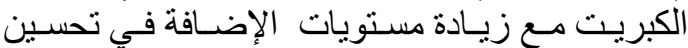

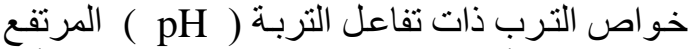

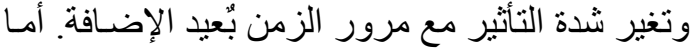

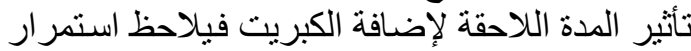
حصول انخفاض في درجة تفاعل التربة بعد مُدَّة شهر الأنهر

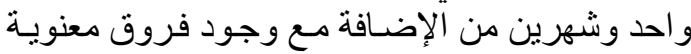

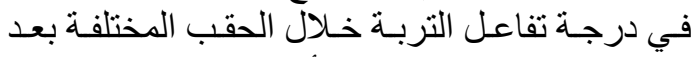

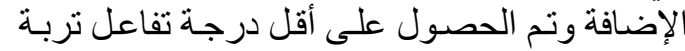

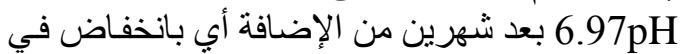

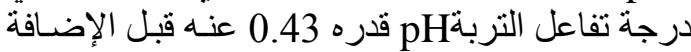

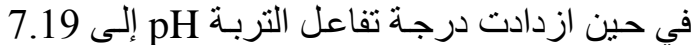

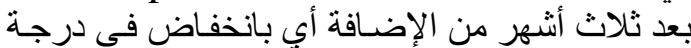

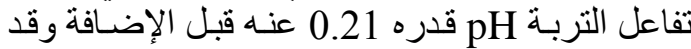

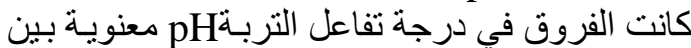

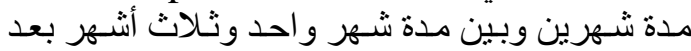

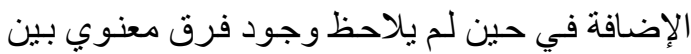

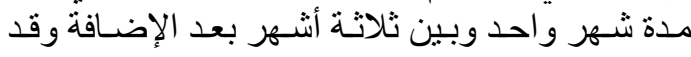

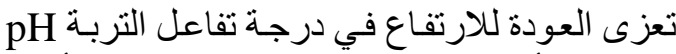

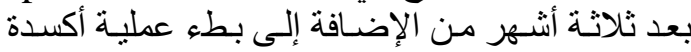

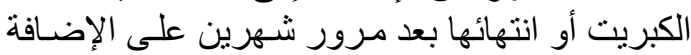

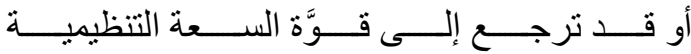
Buffering Capacity

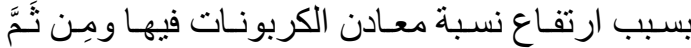

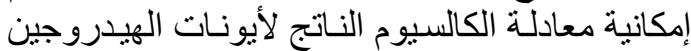

الناتجة من عملية أكسدة الكبريت ( 1 ( ).

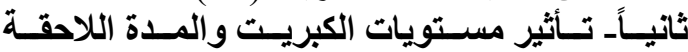

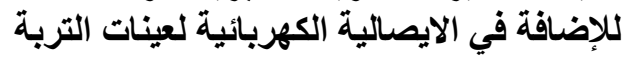

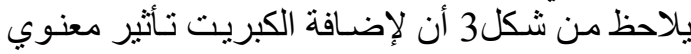

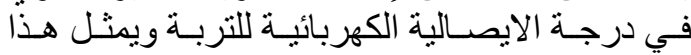

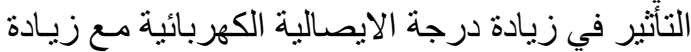

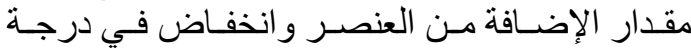

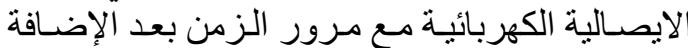

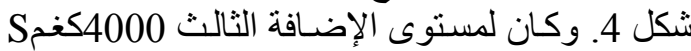

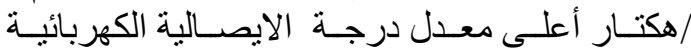

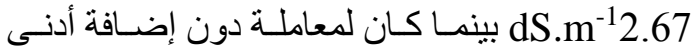

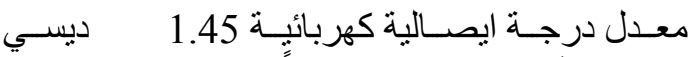

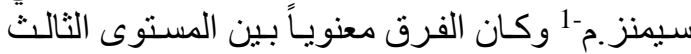

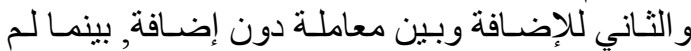

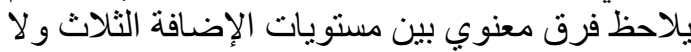

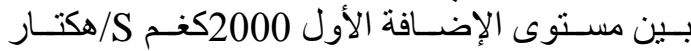

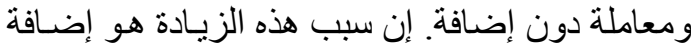

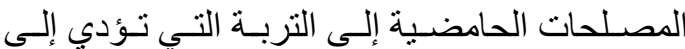

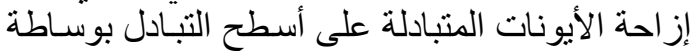
الهيدروجين فتحصل الزيادة بهذه الأيونات في محلول الكادل

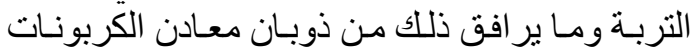




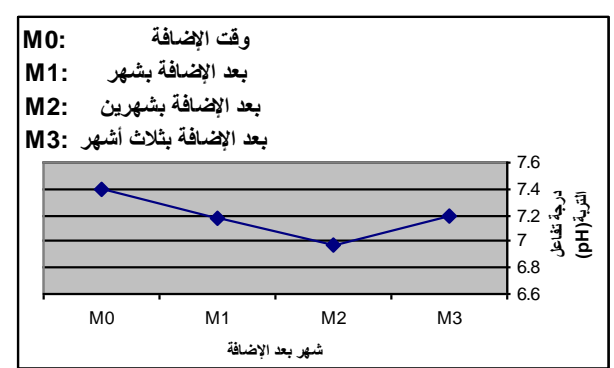

L.S.D \%5 0.12

شكل(2):نأثير الفترة اللاحقة لإضافة الكبريت في درجة تفاعل التربية

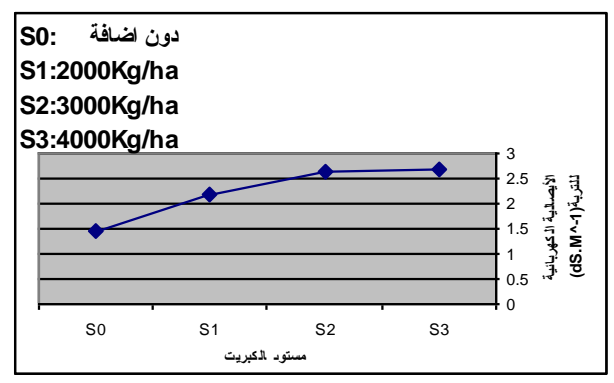

L.S.D \%5 0.9

شكل (3):نأثير إضافة الكبريت في الإيصالية الكهربائبة للتربة

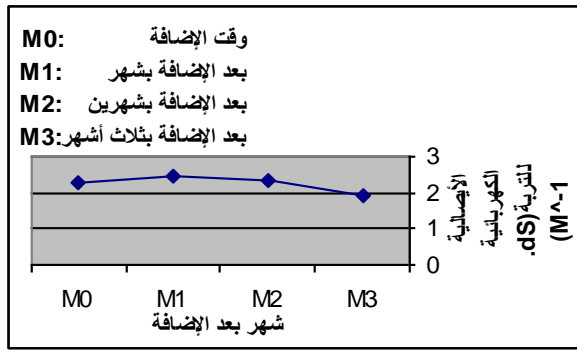

L.S.D $\% 50.21$

شكل(4):تأثير الفترة اللاحقة لإضافة الكبريت في الإيصالية الكهربائية للتربة

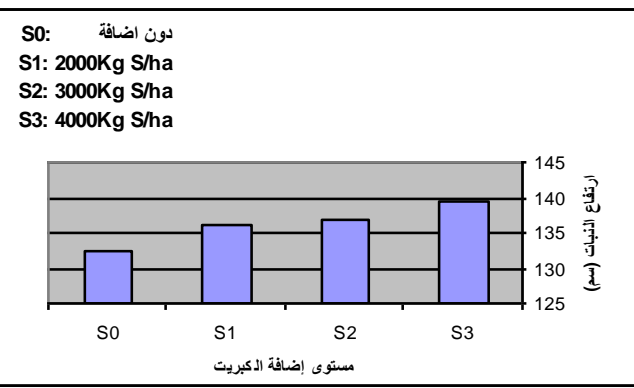

L.D.S\%5 1.14

شكل (5): تأثير الكبريت في معدل ارتفاع النبات

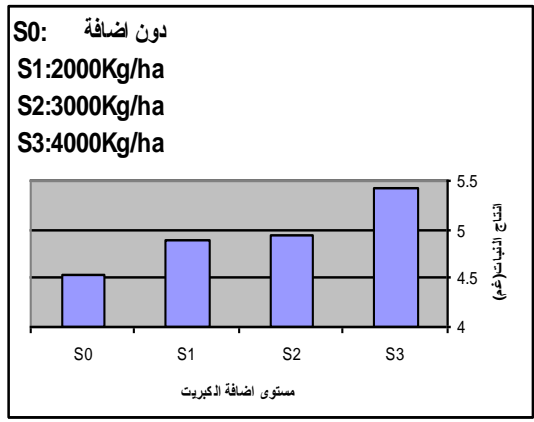

L.S.D 5\% n.s

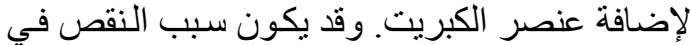

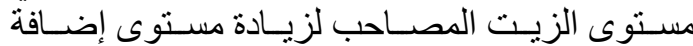

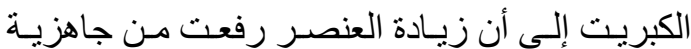

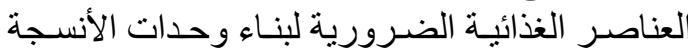

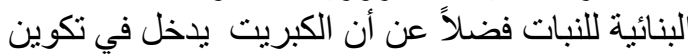

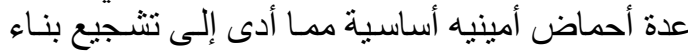

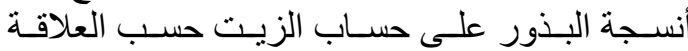
العكسية المعروفة وبالتالي انخفاض نسبة الزيت في

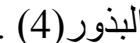

جدول 1 بعض الصفات الفيزيائية والكيميائية للتربة قبل إضافة الكبريت للعام 2005-2004

\begin{tabular}{|c|c|c|}
\hline \multirow[b]{2}{*}{ وحدة القياس } & \multirow[b]{2}{*}{ القيمة } & الصفة \\
\hline & & مفصولات \\
\hline غم. كغم-1 تربة & 184 & طين Clay \\
\hline غم. كغم-1 تربة & 190 & غرين Silt \\
\hline غم. كغم-1 تربة & 626 & رمل Sand \\
\hline \multicolumn{2}{|c|}{$\begin{array}{l}\text { مزيجه رملية } \text { Sandy loam } \\
\text { Sand }\end{array}$} & $\begin{array}{c}\text { نسجة التربة } \\
\text { Soil Texture }\end{array}$ \\
\hline ديسي سيمنز . م-1' & 7.4 & $1: 1 \mathrm{pH}$ \\
\hline ديسي سيمنز . م-1 & 2.3 & $\begin{array}{c}\text { Ece } \\
\text { العجينةة }\end{array}$ \\
\hline غم. كغم-1 تربة & 145 & O.M \\
\hline ملغم. كغم-1 تربة & 16.3 & $\mathrm{~N}$ \\
\hline ملغم. كغم-1 تربة & 13.0 & $\mathrm{P}$ \\
\hline ملغم. كغم-1 تربة & 13.5 & $\mathrm{~K}$ \\
\hline سنتيمول. كغم-1 تربة & 7.4 & $\mathrm{So}^{-2}$ \\
\hline سنتيمول. كغم-1 تربة & 8.2 & $\mathrm{Cl}^{-1}$ \\
\hline سنتيمول. كغم-1 تربة & 7.6 & $\mathrm{Na}^{+1}$ \\
\hline سنتيمول. كغم-1 تربة & 2.96 & $\mathrm{Mg}^{+2}$ \\
\hline سنتيمول. كغم-1 تربة & 5.04 & $\mathrm{Cu}^{+2}$ \\
\hline
\end{tabular}

جدول(2) بعض الصفات للكبريت الزراعي المستعمل في الدراسة.

\begin{tabular}{|c|c|c|}
\hline وحدة & القيمة & الصفة \\
\hline القياس & 3.9 & $\mathrm{pH}$ \\
\hline ديسي سيمنز .م-1 & 4.8 & Ec \\
\hline$\%$ & 95 & الكبريت \\
\hline $\mathrm{ppm}$ & 62 & الكالسيوم \\
\hline$\%$ & - & الكلس \\
\hline$\%$ & 0.12 & الكاربون \\
\hline$\%$ & 1.4 & الطين \\
\hline Mesh & 320 & القطر \\
\hline
\end{tabular}

دون اضافة:S0

$\mathrm{S} 1: 2000 \mathrm{Kg} \mathrm{S} / \mathrm{ha}$

S2:3000Kg S/ha

S3:4000Kg S/ha

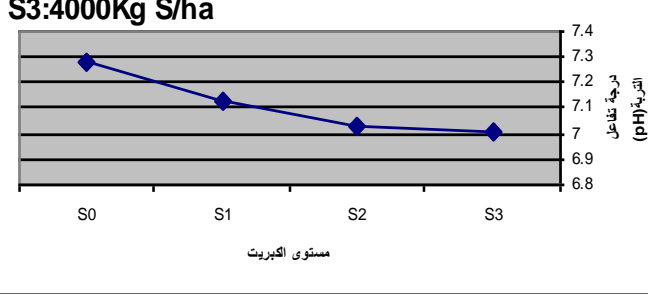

L.S.D \%5 ، 0.13

شكل (1):تأثير إضافة الكبريت في درجة تفاعل التربة 
sulfur by products and their affection characteristics of calcareous soils. Mesopotamia. J Agric. N: 87-95.

12. Aulakh, M.S.; N.S. pasricha and N.S. Sahota. (1980). Yield, nutrient concentration and quality of mustard crops as influenced by nitrogen and sulphur fertilizers.J.agric.Sci., Camb. , 94:545-549

13. Bennett, R.N.and R.M.Wallsgrove. (1994). Secondary metabolites in plant defense mechanisms. New Phytol. 127:617-633.

14. CETIOM. (1996).Rapeseed,Edition center technigue nterprofe ssionaldes oleagineux metropolitains, France.

15. Coleman,Russell.(1966). The importance of sulphur as a plant nutrient in world crop production. Soil Science .101(4):230-239.

16. Dawood, F.A. (1980). Sulfur waste materials for calcareous soils acidulation.Ph.D.Dissertation,Univ of Arizona Tucson, AZ. U.S.A.

17. Ernst, W. H. O.(1993). Ecological aspects of sulfur in higher plants: Agricultural and Environmental Asspects. Eds. L J De Kek. I stolen. H Rennenberg. C Brunold and W E Rauser. Pp 295-313. SPB Academic Publishing bv. The Hauge.

18. Fenwick G.R.; R.K. Heany and W.J. Mullin. (1983). Glucosinolates and their breakdown products in foods and food plants. CRC Crit. Rev. Food Sci. Nutr. 18:123-201.

19. Fismis, Joelle; Phuy Chhoy Vong and Armand Guckert. (1997). Nitrogen and sulphur nutrition of oilseed rape and crop quality. Laboratoire agronomie et environnement. Plant nutrition. Plant nutrition for sustainable food production and environment,Tokyo, Japan. :843-844

20. Janzen, H. H. and J.R. Bettany. (1984).Sulphur nutrition of rapeseed: I. Influence of fertilizer nitrogen and sulfur rates. Soil Sci. Soc. AM. J. 48:100-107.

21. Khan Noorullah; Amanullah Jan; Ihsanullah; Ijaz Ahmed Khan and Naeem Khan. (2002). Response of canola to nitrogen and sulphur nutrition.Asian journal of plant sciences.1(5):516-518( انترنت )

22. Kullman, A. (1991). Reaction pods of oil seed rape (Brassica napus L.) in Relation to raceme position and Nitrogen supply. University of Kiel, GCIRC 1991. Congress, P. 607-611.

23. McGrath, S.P. and F. J. Zhao. (1995). A risk assessment of sulphur deficiency in cereals using soil and
شكل (6): تأثير الكبريت في انتاج النبات من البذور

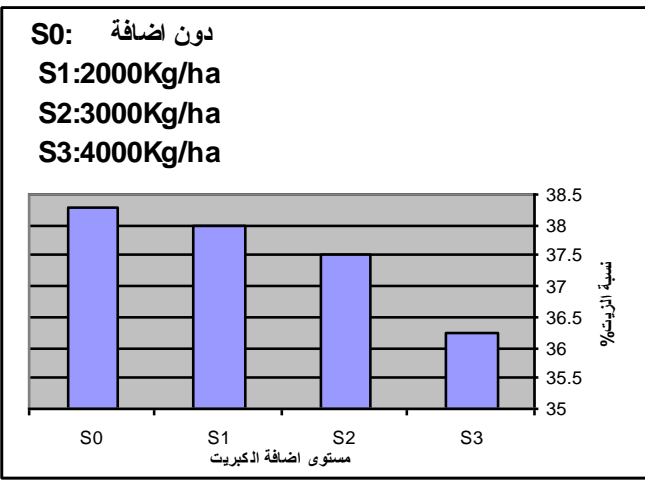

L.S.D 5\% 0.89

شكل (7): تأثثر الكبريت في نسبة زيت البذور

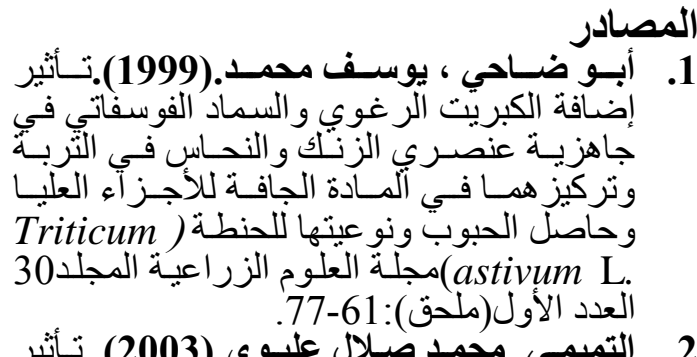

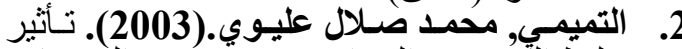

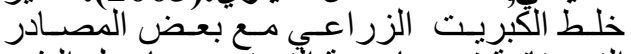

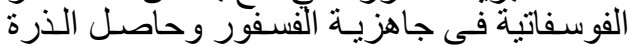

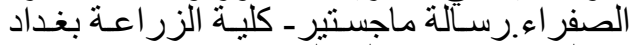

رسالة ماجسنتير - كلية الزر اعة أنس - جامعة بغداد.

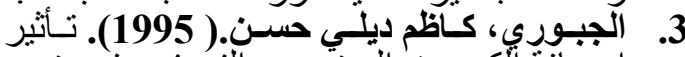

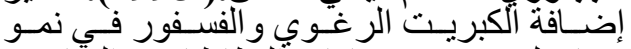

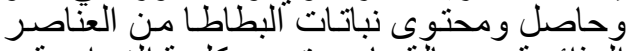

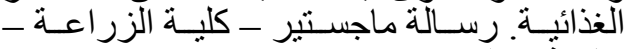
جامعة بغداد.

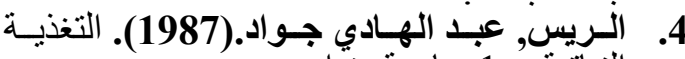

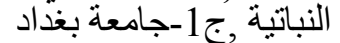

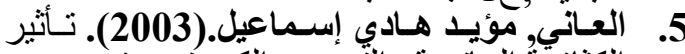

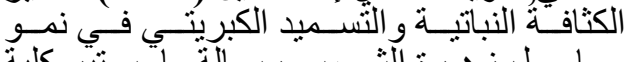

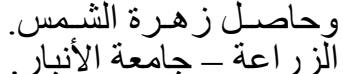

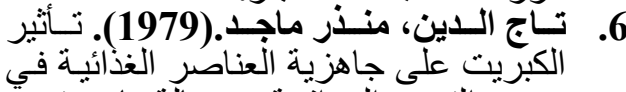

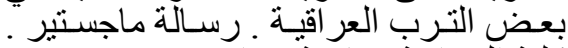

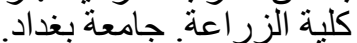

7. شأكر ، عبد الؤهاب عبد الزرزاق ـ 1996. تأثنير

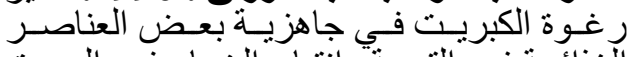

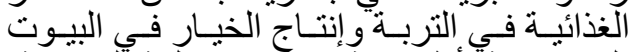

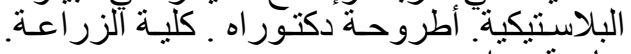

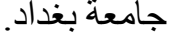

8.Aldric,D.G. Turrel,F.M. (1960) Effect of soil acidification on some chemical properties of soil and the plants grown there on.Soil Sci.70:83-90

9.Admzyk, 2., M. Winia rska, and Kobus. (1975). Microbal oxidation of elemental sulphur in brown soils. Plant and soil. 3: 93-100.

10.Al-Ani, F., M. Abd-lga Wad, and T. Naji. (1977). Iron and phosphorus availability in soil and barley yield as influenced by sulfur application. Iraqi J. Agric. Sci. 12: 124-136.

11.Al-Jubouri, K.D.; E.M. Kalifa; and 1.M. Hajhim. (1976). Application of 
25. Pepper,I. and E. Miller.(1978).Comparison of the oxidation of thiossulfate and elemental sulphur by two heterotrophic bacteria and Thiobacillus thio oxidane.Soil Sci.126: 9-14.

26. Venima,R.C.W.(1962).Some notes regarding the function of sulphate anion in the metabolism of oil producing plants especially oil palm. Pot-

ash in tropical agriculture 5(4):40-43 atmospheric deposition data. Soil uses manage. (11):110-114.

24. McGrath,S.P.;F.J. Zhao and P.J. A.Withers.(1996). Development of sulphur deficiency in crops and its treatment. Proceeding of fertilizer society.No.379.the fertilizer society. Peterborough.

\title{
Effect of sulphur on soil pH, growth and productivity of rape seed plant
}

\author{
Ali. M.A. Al-Khafage*, Laith Mohammed Jawad Al-Shamma**
}

\footnotetext{
*General Board Date Palm/ Ministry of Agriculture

** Biology.Dept/College of Science/University of Baghdad
}

\begin{abstract}
A field trial was conducted in Experimental Station of The Field Crops Department - College Of Agriculture In Abu Ghraib, University of Baghdad to assess the effect of sulphur applications and the time after application on $\mathrm{pH}$ and $\mathrm{EC}$ of soil sample solutions ,and on the growth and yield of rape seed (Brassica napus L.)A split plot design was used with four replications , The main plot included four sulphur applications levels $(0,2000,3000,4000 \mathrm{Kg} \mathrm{S} / \mathrm{ha})$ the sub plot were the time after application $(0,1,2$, and 3 moths) .Sulphur application significantly decreased soil $\mathrm{pH}$ value ,although that decrease reached minimum parameter after two months from application date .Rather increment of sulphur application level significantly raised soil EC value, So that value decreased continuously with time after the application. Sulphur application level affected significantly increased plant height $/ \mathrm{cm}$ and decreased seed oil content percent but not affected in seed yield /plant.
\end{abstract}

